\title{
Analysis of Abrasives On Cutting Edge Preparation By Drag Finishing
}

\section{Dejin Lv}

Shanghai University

\author{
Yongguo Wang ( $\nabla$ ygwang@shu.edu.cn ) \\ Shanghai University \\ Xin Yu \\ Shanghai University \\ Han Chen \\ Shanghai University \\ Yuan Gao \\ Shanghai University
}

\section{Research Article}

Keywords: Drag finishing, abrasive medium, K-factor, kinematics trajectory, cutting edge micro-geometry

Posted Date: July 9th, 2021

DOI: https://doi.org/10.21203/rs.3.rs-669967/v1

License: (c) (i) This work is licensed under a Creative Commons Attribution 4.0 International License. Read Full License

Version of Record: A version of this preprint was published at The International Journal of Advanced Manufacturing Technology on January 7th, 2022. See the published version at https://doi.org/10.1007/s00170-021-08623-w. 


\title{
Analysis of abrasives on cutting edge preparation by drag finishing
}

\author{
Dejin Lv, Yongguo Wang*, Xin Yu, Han Chen, Yuan Gao
}

School of Mechatronics Engineering and Automation, Shanghai University, Shanghai City, 200072, PR China

\begin{abstract}
Cutting edge preparation has become more important for tool performance. The micro-shape, radius and surface topography of the cutting edge plays a significant role in the machining process. The cutting edge of solid carbide end mills have some micro-defects after grinding. For eliminating aforementioned problem, this study investigates drag finishing (DF) preparation for solid carbide end mills reconstruct cutting edge micro-geometry. This paper is to present the design of DF experimental set-up and analysis the characterization of various abrasive media (K3/600, K3/400, HSC $1 / 300$ and HSO 1/100) on the evolution of the surface /roughness along the cutting edge. In parallel, the mechanism of material removal and the kinematics trajectory of the drag finishing are presented. In fact, the form factor (also called as "K-factor") of the cutting edge micro-geometry is quantified. Comparing with four lapping media, the higher material removal rate (MRR) and the lower surface roughness are obtained by HSO 1/100 abrasive process. The results show that the cutting edge K-factor, MRR and surface topography are influenced by the abrasive particles size, composition and process time. The cutting edge micro-geometry is measured through Scanning Electron Microscopy (SEM) and 3D Optical measuring instrument.
\end{abstract}

Keywords: Drag finishing; abrasive medium; K-factor; kinematics trajectory; cutting edge micro-geometry

\section{Introduction}

Cemented carbide tools have higher hardness and higher toughness, and have good temperature wear properties, suitable for metal materials in recent decades. Solid carbide end mills have a complex helical surface, and the irregular cutting edge take shape after grinding. Therefore, cutting edge preparation is an indispensable manufacturing process in the tool grinding process. L i et al. [1] analyzed the grinding process of helical groove of the solid carbide end mill, and pointed out that the grinding parameters play an important role in the geometry of the end mills. Denkena et al. [2] studied the influence of cutting edge microscopic geometry on tool wear and life. The results show that the flank face wear and the cutting edge rounding are positively correlated in machining AISI4140, where the state of art cutting edge micro-geometry can be determined according to the cutting parameters. Bouzakis et al. [3] reviewed the influence of cutting edge preparation on coated tools. The result was shown that the cutting performance improvement was obtained almost by all edge preparations.

Solid carbide end mills composed of carbide and cobalt, high brittleness, formation of micro-cracks, micro-chipping and sharp cutting edge after grinding. Karpat et al. [4] reported that the sharp cutting edges were generally considered to be unfavorable for the cutting process because of their low stability and poor impact resistance. The preparation of solid carbide end

Corresponding author.

E-mail addresses: ygwang@shu.edu.cn 
mills has become an indispensable process, in order to reduce edge chipping at the beginning of the cutting process, increasing tool life and process reliability. Denkena et al. [5] reviewed several studies on the cutting edge preparation methods and summarized cutting edge micro-geometry how to influence the whole of cutting process.

Drag finishing (DF), mainly used for deburring, cutting edge preparation and high-demand polishing [6]. Uhlmann et al.[[7]-[8]] conducted experimental study the cutting edge preparation of micro-milling tools using DF method, the results obtained the cutting edge radius range from $4.0 \mu \mathrm{m}$ to $31.2 \mu \mathrm{m}$. The crucial factors affecting the cutting edge preparation is the abrasive media. The typical motion of DF is the secondary planetary motion. The removal of material mainly depends on the relative movement between the abrasive and the tool. During the second planetary motion, Gillespie et al. [9] and Holzknecht et al. [10] were measured the final surface quality and material removal rate (MRR) by the abrasive dynamics and the initial surface roughness of the workpiece. Nguyen et al. [11] was studied Silicon carbide ( $\mathrm{SiC}$ ) abrasive, which has stable chemical properties, high thermal conductivity, low thermal expansion coefficient, good wear resistance, and can be used as abrasive medium. Quartz, the main chemical component of is $\mathrm{SiO}_{2}$, which is an inorganic substance with very stable physical and chemical properties. Lee et al. [12] used silica $\left(\mathrm{SiO}_{2}\right)$ chemical mechanical polishing technology to establish a material removal rate distribution model. The analysis included particle size, particle concentration, slurry flow rate, density of the abrasive pad surface and chemical reaction. Abrasives were usually in the form of hard mineral particles, which removed material by generating large local stresses and strains on the surface of the part. Pellegrin et al. [13] characterized the shape and scale of complex particles, using Euclidean three-space established the abrasion model. Uhlmann et al. [14] used two different lapping medias (HSC 1/300 and H4/400) experimental investigations on the preparation of four tool groups of micro end mills cutting edge geometry, the cutting edge radius $r_{\beta}$ and the cutting edge chipping possibility to control by the processing time $t_{B}$. Sooraj et al. [15] dedicated the important issues of cutting edge preparation about abrasive media, due to the complex cutting tools and fixtures, cost-effectiveness, and surface finishing.

Cutting edge preparation is the interaction between the solid particles and the workpiece in the slurry, leading to mechanical removal material from the surface of the workpiece. Mechanical aspects of the interaction of a granule and a tool surface have been widely studied. Evans et al. [16] reviewed the lapping and polishing process, and analysized the solid granules physical removal material mechanism. Barletta et al. [17] established the basic mechanisms of solid particle removal ductile metal workpiece material through fluidized bed assisted drag finishing. Sundararajan et al. [18] defined the collision process between abrasive and workpiece as the hypothesis of localization of plastic deformation. Brach et al. [19] according to the Hertzian contact theory defined the erosion of ductile metal as the transformation from the collision energy of solid particles to internal deformation energy. Mechanical aspects of the interaction of a granule and a workpiece surface have been widely studied. Yang et al. [20] conducted experimental study the alumina abrasive and garnet abrasive about the abrasive flow velocity and energy distribution in cutting performance. Fowler et al. [21] reported that, the abrasive media, size, shape factor have significant influence the tool cutting performance. With increasing particle hardness, the material removal rate and surface roughness increased. Only by choosing the right medium can the desired cutting edge micro-geometry and topography. Malkorra et al. [22] researched on the mechanism of material removal aim to provide answers to the following questions: how surface topography 
formation influenced by different abrasive processes, process parameters such as processing time $t_{B}$ and rotation speed, commercially available abrasive media and the initial surface roughness $R z$ of workpiece.

This article aims to provide a better understanding of mechanical removal mechanisms, with special emphasis on the role of abrasive media. The correct choice of abrasive media is the key to the success of obtaining uniform cutting edge micro-geometry and topography. Hopefully, this paper will help provide some guidance on the tailored cutting edge preparations. According to experiments, most of the available research is deterministic and/or statistical, but there is less experience and less understanding of material removal and motion trajectory issues. Consequently, this paper presents a detailed drag finishing preparation process with four-kind abrasive medium in the mechanism material removal rate and the investigate the surface topography by SEM.

\begin{tabular}{|llcll|}
\hline Nomenclature & & \\
$K$ & From-factor & $r_{\beta}$ & Cutting edge radius \\
$S \gamma$ & Cutting edge segment on the rake face & $r_{R}$ & Sun wheel radius \\
$S \alpha$ & Cutting edge segment on the flank face & $r_{H}$ & Planetary wheel radius \\
$A \gamma$ & Rake face & $\bar{S}$ & Cutting edge rounding \\
$A \alpha$ & Flank face & $\beta$ & Cutting edge wedge \\
$\varphi$ & Apex angle & $A_{r}$ & Material removal area \\
$d_{G}$ & Abrasive grain diameter & $\theta$ & Rotation angle \\
$n_{R}$ & Rotational speed of the first-planetary motion & $r_{s}$ & Initial cutting edge radius \\
$n_{H}$ & Rotational speed of the first-planetary motion & $\Delta r$ & Profile flattening \\
\hline
\end{tabular}

\section{Materials and methods}

\subsection{Abrasives}

In the process of cutting edge preparation, Steigerwald et al. [23] investigated the abrasive in the slurry play a very important role in transferring mechanical energy to the machined surface. In terms of metal cutting, the tools are used as industrial teeth. From the perspective of cutting edge preparation, abrasive media can be understood as the teeth of the tool. They have no specific geometric cutting edges, and can be distinguished by the high hardness with sharp edges and also having good cutting abilities. Holzknecht et al. [24] were good description of the sharpness of abrasive particles including roundness and apex angle. Nie et al. [25] obtained the abrasive roundness model by enlarging the shape according to the estimation method in the literature. As the size of abrasive particles increased, the sharpness of the apex angles of these particles decreased, which reduced the cutting ability of the abrasives.

The abrasive of drag finishing with four different medium, namely K3/600, K3/400, HSC 1/300 and HSO 1/100 supplied by OTEC, Germany. The abrasive particles are observed by magnifying 100 as shown in Fig. 1. K 4/600 and K 4/400 abrasives are mainly composed of walnut shells, which are widely used because of its high cost performance and relatively low wear and different sizes of walnut shells define different specifications. The above abrasives are all added with polishing paste containing diamond particles (in the range of a few microns), which are designed for rapid finishing and removal of large amounts of material. The HSC 1/300 abrasive in the experiments consists of $30 \%$ silicon carbide ( $\mathrm{SiC}$ ) and $70 \%$ walnut shell granulate of K3/400 widely used as cutting edge preparation. The abrasive of HSO 1/100 mainly consists of two components $50 \% \mathrm{SiO}_{2}$ and $50 \%$ walnut shell granulate of $\mathrm{K} 3 / 400$, its high hardness and 
material removal have unique performance in the cutting edge preparation process, which is also an important research in this paper. The physical properties of abrasive media are shown in Table 1. To compare the differences in mechanical properties with abrasive during slurry, twenty end mills were prepared with each abrasive for five end mills.
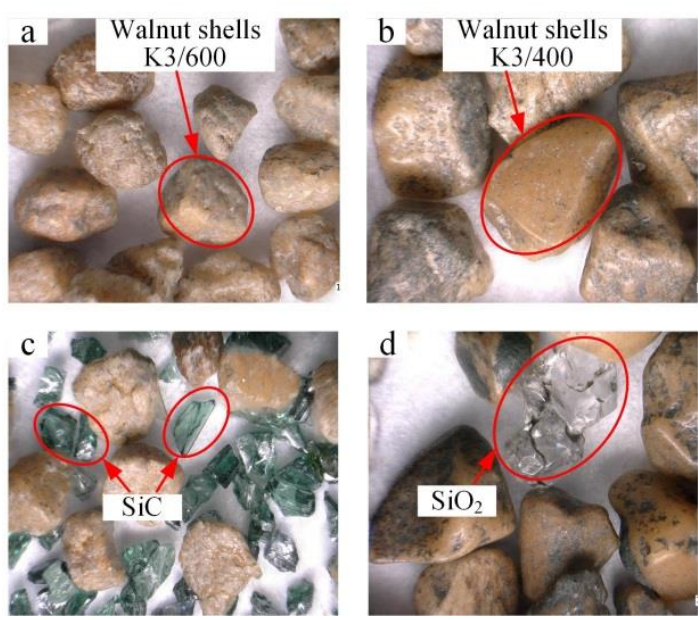

Fig. 1 Microscope images of abrasive particles

Table 1 Abrasive medium used for drag finishing

\begin{tabular}{lcccc}
\hline Analysis typical & $\mathrm{K} 3 / 600$ & $\mathrm{~K} 3 / 400$ & $\mathrm{SiC}$ & $\mathrm{SiO}_{2}$ \\
\hline Hardness $\left[\left(\mathrm{kg} / \mathrm{mm}^{2}\right]\right.$ & 1500 & 1500 & 2700 & 2400 \\
Density $\left[\mathrm{g} / \mathrm{cm}^{3}\right]$ & 3.0 & 3.0 & 3.2 & 3.6 \\
Average diameter of grain $[\mathrm{mm}]$ & $0.4 \leq d_{G} \leq 0.8$ & $0.4 \leq d_{G} \leq 0.8$ & $0.4 \leq d_{G} \leq 0.8 \& d_{S c} \approx 0.2$ & $0.8 \leq d_{G}, d_{S o} \leq 1.3$ \\
\hline
\end{tabular}

\subsection{Cutting tool}

Solid carbide end mills are produced by a ANCA TX7 +5 -axis CNC grinding machine. The 3D model of end mill is derived from the tool room grinding simulation software by ANCA as shown in Fig. 2a. After grinding, the marks and irregular defects are significantly affect the tool life. Irregular cutting edges micro-geometry increase the stress concentration in the cutting process, leading to premature failure. Weinert et al. [26] investigated the grinding marks how to influence the chip flow, and the higher friction temperature and chip compression were observed. Lv et al. [27] reported that the size of W-Co grains correlated with a high degree of edge chipping, and the tool substrate of cobalt proportion result into the cutting edge roundness. In this paper, the uncoated solid carbide end mills with $8 \%$ Co and average grain size of WC particles was measured to be about $0.6 \mu \mathrm{m}$ was shown in Fig. 3. These end mills are in four flute, six-diameter with hardness $\mathrm{HV}=1.7 \mathrm{kN} / \mathrm{mm}^{2}$, supplied by Guohong tool system (Wuxi) Co., Ltd. The milling tool geometric parameters were shown in Table 2.

The cutting edge micro-geometric characteristics are shown in the Fig. 2b. Zhou et al. [28] reported that edge radius $r_{\beta}$ is normally defined the rounded cutting edges. But the micro-shape of cutting edge is actually not constant circle and cannot be characterized by only one parameter. Denkena et al. [5] established more precise characterization method with four parameters $\mathrm{S} \gamma, \mathrm{S} \alpha$, $\Delta \mathrm{r}, \varphi$ are presented in Fig. $2 \mathrm{~b}$. It is defined as the form factor method. 


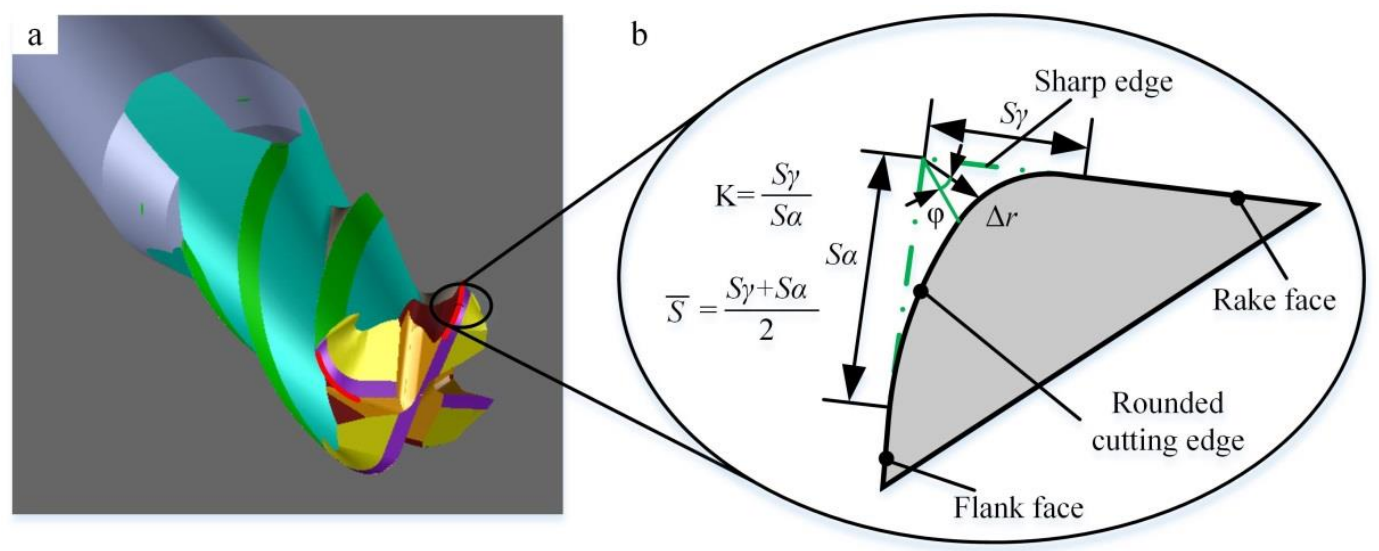

Fig. 2 (a) Solid carbide end mill (b) K-factor of cutting edge characterization

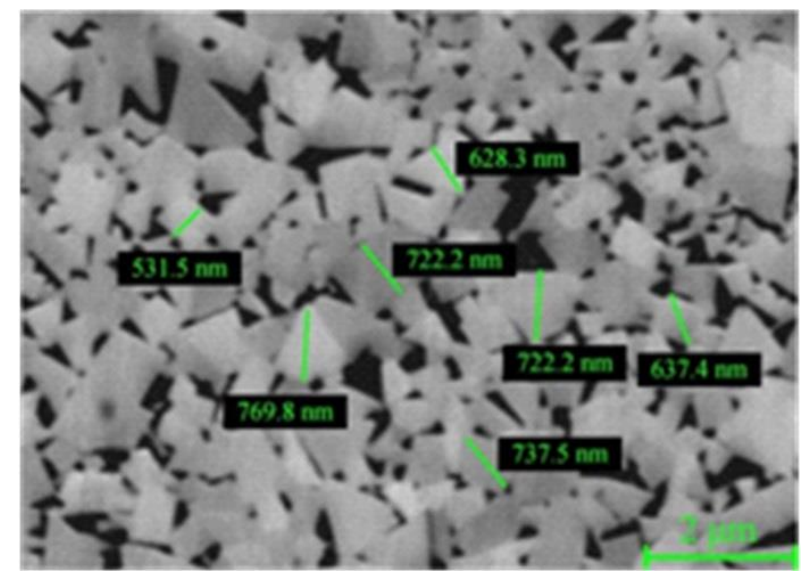

Fig. 3 The grain size of Cemented carbide milling cutter

Table 2 Solid carbide end mills geometric parameters.

\begin{tabular}{ll}
\hline Parameter & Value \\
\hline Shank diameter $D$ & $8 \mathrm{~mm}$ \\
Tool diameter $d$ & $6 \mathrm{~mm}$ \\
Number of teeth $Z$ & 4 \\
Tool length $L$ & $55 \mathrm{~mm}$ \\
Cutting edge length $l$ & $10 \mathrm{~mm}$ \\
Helix angle $\beta$ & $40^{\circ}$ \\
Radial rake angle $\gamma$ & $0^{\circ}$ \\
Radial relif angle $\alpha$ & $8^{\circ}$ \\
Corner radius $R$ & $1 \mathrm{~mm}$ \\
\hline
\end{tabular}

\subsection{Drag finishing machine and parameters}

For the cutting edge preparation of solid carbide end mills with rounding cutting edge, drag finishing (DF) is an appropriate process. The DF machine was shown in Fig. 4a. DF-3 machine was made by the company OTEC PRÄZISIONSFINISH GMBH, Straubenhardt, Germany. In this drag finishing process, the milling tools fixed on holders and immersed in an abrasive lapping medium. From the Fig. 4a, the tools move along the two-stage planetary motion trajectory through the abrasive particles. The ability of material removal is the result of the relative movement between the cutter and the abrasive. The spindle motor turns clockwise (red arrow) to drive the 
coupling, causing the planetary motion to revolve in the same direction as the spindle motor. As a result, the rounded cutting edge is prepared with the abrasive in both rotation $n_{R}$ and revolution $n_{H}$.

The experimental analysis focuses on the cutting edge preparation by abrasive medium $\mathrm{K} 3 / 600, \mathrm{~K} 3 / 400$, HSC $1 / 300$ and HSO $1 / 100$, which enhance MRR on the cutting edge, it can also achieve more efficient deburring and micro-defect. During the grinding process surface quality, surface characteristics and surface texture of rake and flank face play an essential role in solid carbide end mills performance. As Fig. 4c, the surface of the rake and flank face are obvious traces of the grinding wheel and micro-defect are obvious near the cutting edge. After preparation by HSO 1/100, the micro-defect and grinding lines disappeared as shown in Fig. $4 \mathrm{~d}$.

All of the applied end mills have been produced out of one production batch with uncoated in Fig. 4b. The abrasives were listed in Fig. 1. The prepared cutting edges were labeled as $E i$, where " $i$ " depends on the abrasive medium indices for all prepared end mills. The unprepared solid carbide end mills E0 have an average cutting edge radius of $r_{\varepsilon}=3.35 \pm 0.58 \mu \mathrm{m}$. For the investigations of four tool groups, each with five end mills were formed and each group has an abrasive preparation. The process parameters are given in Table 3. For all preparation parameters, the direction of rotor motion and the tools is clockwise for 1 minute and counterclockwise for 1 minute, and the total processing time is 20 minutes.

Table 3 Process parameters for drag finishing.

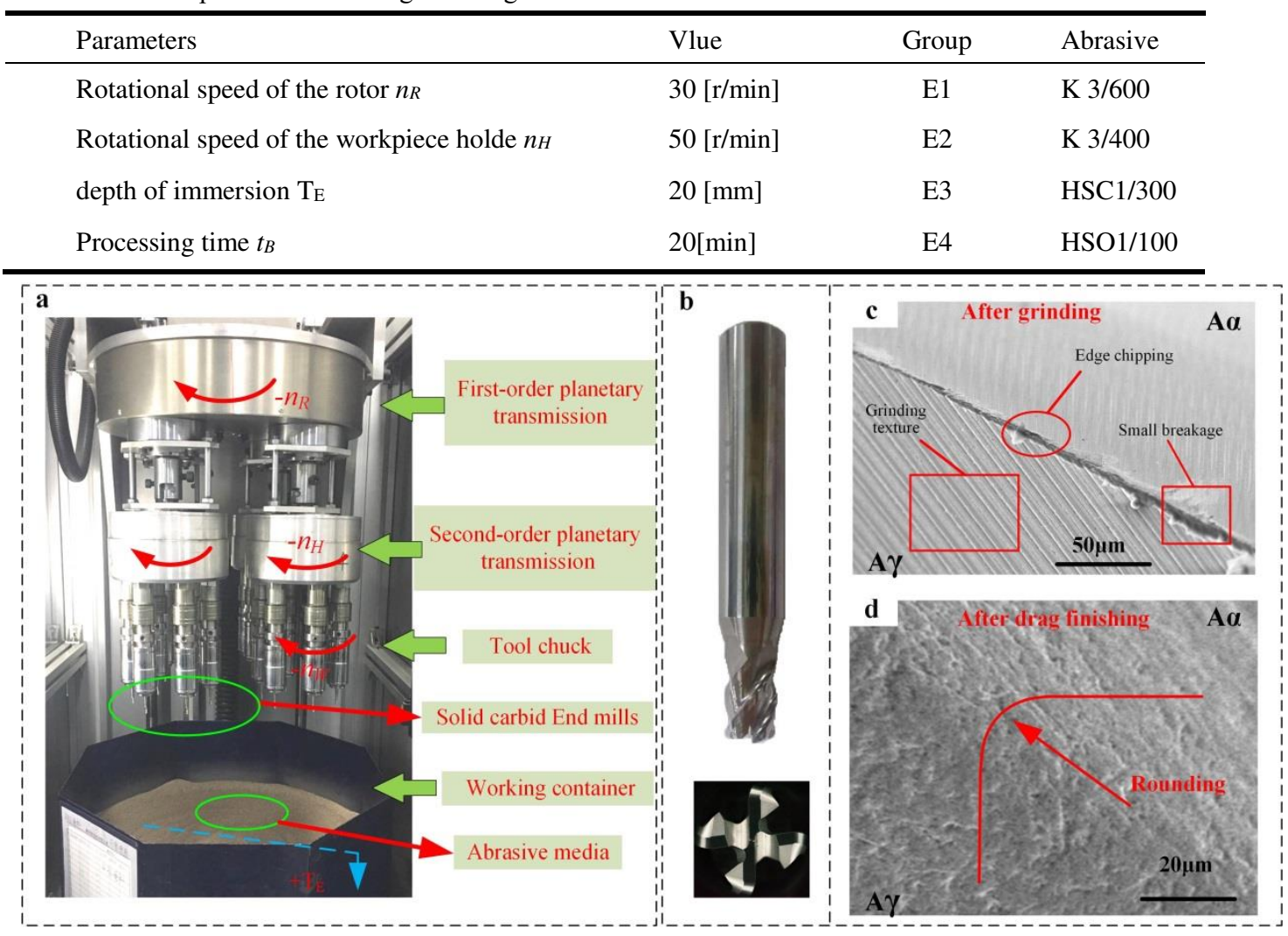

Fig. 4 DF process (a) DF machine (b) solid carbide end mills (c) cutting edge micro-defect after grinding (d) cutting edge rounding after HSO 1/100 abrasive preparation

\subsection{Cutting edge measurement}

The edge geometry of all prepared solid carbide end mills are measured with Infinite-Focus G5 microscope by ALICONA. The cutting edge micro-geometry was measured by built-in Edge Master Module and through the 50 edge profiles. As Fig. 5, the green lines illustrates the fitting 
data. The four cutting edges of each tool are measured, and the average value is calculated and recorded. The entire cutting edge preparation process have a maximum deviation of $10 \%$ from the specified nominal rounding as mentioned by [2].

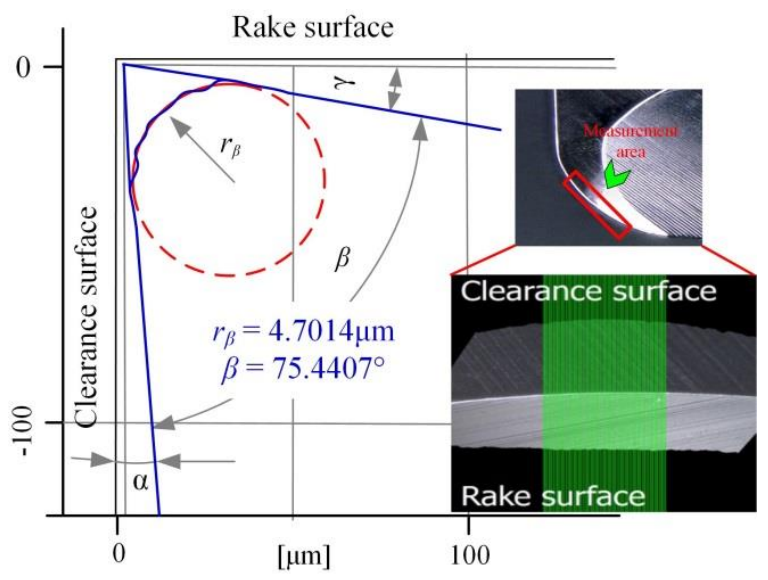

Fig. 5 Cutting edge measurement using Alicona microscope

The cutting edge surfaces were optically scanned measuring instrument by Alicona G5. The cutting edge chipping measurement is automatically set along the cutting edge in the Edge master module. Cutting edge chipping and roughness precise measurement along the cutting edge. Moreover, some measurements with physical surface quality (arithmetic average height $R a$, total height of profile peak and valley $R t$ and ten-point height $R z$ ) have also obtained by Edge master module. The value $R t$ reflects the largest chipping of the cutting edge. Solid particle abrasion and surface roughness analysed on the cutting edge after process $20 \mathrm{~min}$. Composed by five roughness profiles each, were performed on each sample, make sure the roughness profiles to be fixed in one positions. Thus, ensures the same position of the each cutting edge after drag finishing, Ricardo et al. [29] measured 2D-Roughness by profilometer. Scanning electron microscope (SEM) was used to discover and identify the microscopic topography caused by various abrasive processing on the tool surface.

\section{Results and discussion}

\subsection{Material removal process of $\mathrm{DF}$}

\subsubsection{Analysis of abrasion mechanism}

A basic understanding of material removal mechanisms is essential for developing predictive models and optimizing lapping processes. The function of solid particles in the slurry is to physically remove materials from the surface of the end mill. Beaucamp et al. [30] studied shows that the material removal (MR) model of hard and brittle materials involved brittleness and ductility mechanisms, which depend on abrasive composition. Lin et al. [32] also verified this study. Kato et al. [31] also explained that the process of MR was a complex mechanism because of its nonlinear and dynamic nature. During the movement of the abrasive and end mills, the contact between the particle and the cutting edge will only be established within a short period of time, resulting in impact and energy transfer thereby smoothing the surface. The material removal process largely depends on the mechanical action of the cutting edge and particles, as mentioned by Hutchings et al. [33] and $\mathrm{Lu}$ et al. [34]. The mechanism of brittle material removal explained in this paper is based on a detailed literature review Chiaia et al. [35]. Typical abrasive cutting and material removal mechanism of drag finishing is shown in Fig. . 


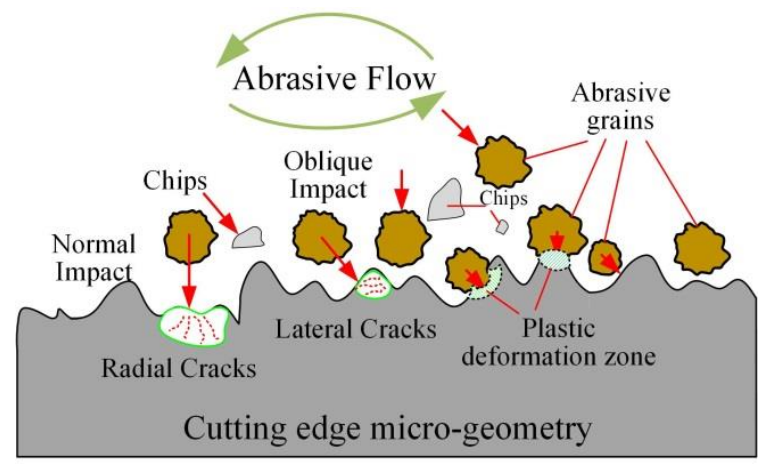

Fig. 6 Schematic illustration of the abrasive process

The impact removal mechanism of loose abrasive media on brittle materials in DF equipment can be divided into two types: brittle fracture and plastic deformation. The former is similar to the indentation of a hard indenter on brittle materials. It involves two main crack systems, in which lateral cracks are responsible for material removal, while radial cracks lead to strength degradation in Fig. 6 the red dotted line position. The latter is similar to the chip formation process in metal grinding, which involves scraping, ploughing and chip formation, as mentioned by [36]. Some assumptions based on the abrasive removal process are as follows

- The abrasive particles are spherical and harder than the workpiece material.

- The movement of abrasive particles will not undergo plastic deformation or brittle fracture.

- No chemical reaction occurs on the particles and cutting edge contact interface.

- The crater produced by the crack propagation and chipping in brittle material is similar hemispherical in shape.

- The processes of adhesion and deformation energy loss are independent .

According to this hypothesis, all materials, regardless of their hardness and brittleness, will undergo a transition from brittleness to ductile processing. Since the mode of deformation (plastic or brittle) depends on the state of stress and not on the magnitude of the stress. It is difficult to understand the local deformation caused by the material near the abrasion zone. The MR is based on the amount of the kinetic energy of the solid particle impinged cutting edge equal to the energy of deformation. Therefore, the deformation energy of the MR can be determined by the incident energy of the solid particles thus, defined as:

$$
I_{E}=0.5 m v^{2} \sin ^{2} \alpha\left(1-e^{2}\right)
$$

where $v$ and $m$ are the impinging particle speed and mass, $\alpha$ the impact angle, and $e$ the restitution coefficient, which, according to Johnson et al. (1985), can be estimated as follows:

$$
e=\frac{1.7 \sigma_{y-w}^{5 / 8}}{v^{1 / 4} \rho_{a}^{1 / 8}\left(E^{*}\right)^{1 / 2}}
$$

where $\sigma_{y-w}$ is the yield strength of the milling cutter, $\rho_{a}$ the abrasive particle density, and $E^{*}$ the equivalent modulus, that is:

$$
\frac{1}{E^{*}}=\frac{1-v_{a}^{2}}{E_{a}}+\frac{1-v_{W}^{2}}{E_{W}}
$$


where $E_{a}$ and $E_{w}$ are the elastic modulus of abrasive particle and milling cutter, and $v_{a}$ and $v_{w}$ are Poisson's ratio Equation (3) provides an estimate of the material removal potential of solid particles during slurring.

When the particle movement has a high and positive strain hardening capability $(\delta \sigma / \delta \varepsilon>0$, $\sigma=$ flow stress, $\varepsilon=$ plastic strain), the plastic deformation beneath the abrasives spreads uniformly for volume energy, as mentioned by Brach et al. [37] and Sundararajan et al. [38]. Conversely, if the kinetic energy of the particle does not exceed the tool material's strain or work hardening capacity (ie $\delta \sigma / \delta \varepsilon>0$ ), the deformation under the particle is immediately confined to the near surface layer, leading to the formation of lip. Under this condition, the material deforms uniformly until the critical strain of $\delta \sigma / \delta \varepsilon=0$. When high than this critical strain, $\delta \sigma / \delta \varepsilon<0$, so the plastic deformation is localized to form a lip. As shown in previous studies [[39]-[40], the plastic flow behavior of materials deformation can be easily obtained, and the critical strain $\left(\varepsilon_{c}\right)$ formed by the lip can be obtained. The following equations are related to flow stress $(\sigma)$, plastic strain $(\varepsilon)$ and temperature $(T)$

$$
\sigma=K \varepsilon^{n}(1-C T)
$$

where $K$ is the strength coefficient, $n$ is the strain hardening exponent and $C$ is a constant characterizing the temperature dependence of the flow stress. The critical strain for localization and hence lip formation $\left(\varepsilon_{c}\right)$ is then obtained as

$$
\varepsilon_{c}=\left(\frac{n \rho_{W} C_{P-W}\left(T_{m} / K\right)}{C T_{m}}\right)^{1 /(n+1)}
$$

where $n$ is strain hardening exponent, $\rho_{w}$ is cutter material density, $C_{p-w}$ and $T_{m}$ are the specific heat and melting point respectively of the abrasive and $C$ is constant which has a value of about 0.5 for many typical metals, as reported by [41].

\subsubsection{Drag finishing kinematics analysis}
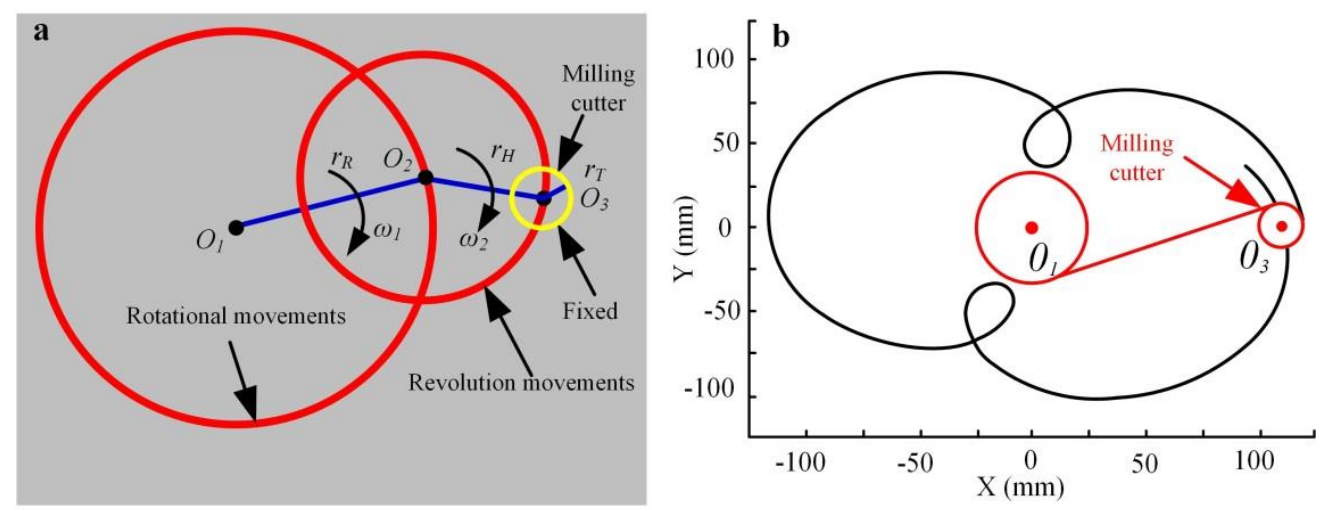

Fig. 7 Drag finishing motion analysis (a) Motion diagram (b) Milling cutter movement trajectory

The drag finishing is a typical equipment for preparing the cutting edge of round bar tools and workpiece polishing as reported by [42]-[43]. The complexity of solid particle abrasion relies on its rotational movement in fixed barrel and the material removal depends on normal and oblique action. When the angular particles are impinged at an oblique angle, the friction between the abrasive and the workpiece will introduce a high level of shear strain in the near-surface area as reference [44]. The dispersed solid abrasive, consisting of K3/600, K3/400, HSC 1/300 and HSO $1 / 100$ in the container. The solid carbide end mills are clamped on the planetary wheel as 
indicated in Fig. 4a, and the cutting edge is prepared by the continuous impact of the loose abrasive particles, which eliminating micro defects and realizing an efficient and uniform cutting edge preparation by drag finishing. The two-stage planetary motion of the tool is shown in Fig. 7a. In Fig. 7a, $\omega_{1}$ is the rotation speed of axis $O_{2}$ around $O_{1}, \omega 2$ is the rotation speed of axis $O_{3}$ around $O_{2}, r_{R}$ is the rotation radius of $O_{2}, r_{H}$ is the rotation radius of $O_{3}$, and $r_{T}$ is the tool rotation radius. The pattern of a generic point on the outer surface of the milling cutter during its motion can be accurately described by the following equations (6):

$$
\left\{\begin{array}{l}
x(\theta)=\left(r_{R}+\left(r_{H}+i\right)\right) \cos \theta-d \operatorname{gcos}\left(\frac{r_{R}+\left(r_{H}+i\right)}{\left(r_{H}+i\right)} \theta\right) \\
y(\theta)=\left(r_{R}+\left(r_{H}+i\right)\right) \sin \theta-d \operatorname{gsin}\left(\frac{r_{R}+\left(r_{H}+i\right)}{\left(r_{H}+i\right)} \theta\right)
\end{array}\right.
$$

With this particular motion, the tools movement trajectory by MATLAB 7.0 was shown in Fig. 7b. An epitrocycloid trajectory ensures the maximum contact uniformity between the abrasive and the tool surface, accordingly, the best performance of the drag finishing.

\subsubsection{Material removal rate (MRR)}

In order to characterize the precision removal process for cutting edge preparation, a parameter can be defined that takes into account the volume of material removed, regardless of the length of the cutting edge, and the number of five tools are processed simultaneously. The nominal removal area $A_{r}$, as a parameter to determine the material remove in the preparation of the cutting edge, is shown in Fig. 8. $A_{r}$ represents the nominal initial radius $r_{s}$ transform final radius $r_{\beta}$ formed to remove the area of material. Considering the quantification of the cutting edge preparation process, an expression to determine the nominal removal area of the material is proposed by [43]. For this expression, the change of radius (from $r_{s}$ to and the wedge angle $\beta$ are considered. The four abrasive removal capabilities are shown in Fig. 9.

The nominal removed area $A_{r}$, can be written as follows:

$$
A_{r}=K_{\beta}\left(r_{\beta}^{2}-r_{s}^{2}\right)
$$

Where $K_{\beta}$ is:

$$
K_{\beta}=\frac{\sin ^{2}\left(90^{\circ}-\frac{\beta}{2}\right)}{\tan \frac{\beta}{2}}-\frac{\pi}{2}\left(\frac{180^{\circ}-\beta}{180^{\circ}}\right)+\sin \left(180^{\circ}-\beta\right)
$$

$K_{\beta}$ is the coefficient as function of the wedge angle $\beta$

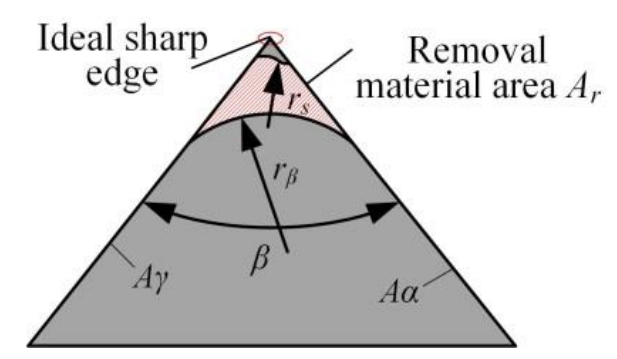

Fig. 8 Concept of nominal removed area $A_{r}$. 


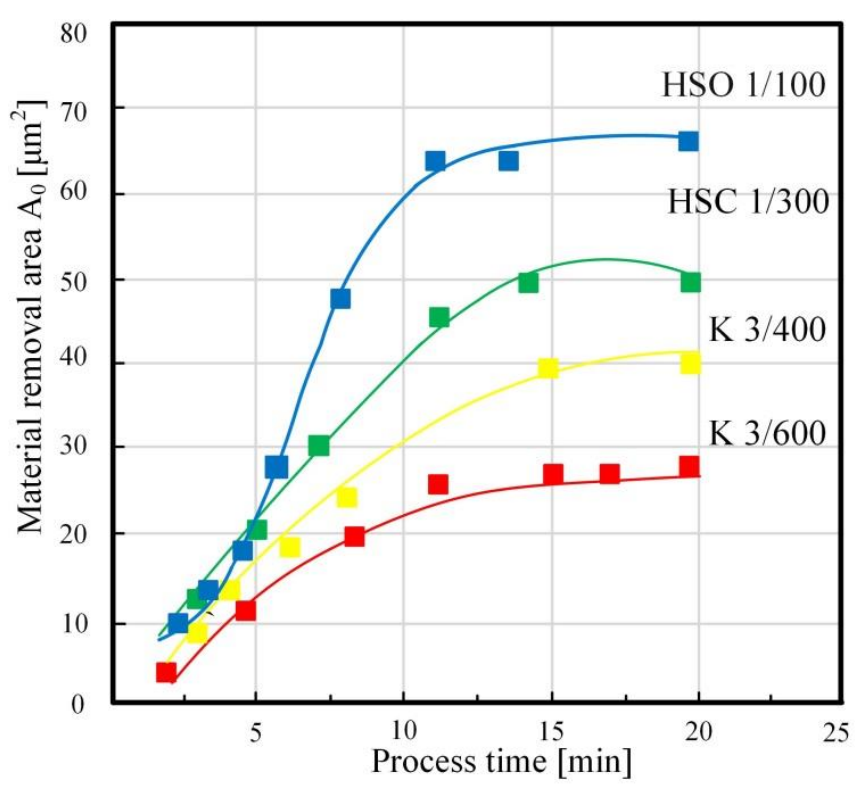

Fig. 9 Material removal area of four abrasives with the time variation

\subsection{Cutting edge characterization}

\subsubsection{Cutting edge $\mathrm{K}$-factor parameters}

The macroscopic and microscopic geometry defines the shape of cutting tool. The micro-geometry characterizes the cutting edge, which is the transition between the rake face and flank face to the cutting wedge. For the rounded cutting edge, the radius $\left(r_{\beta}\right)$ is still a frequently used to characterize the cutting edge micro-geometry. Generally, the fitting shape of the cutting edge is not circular, and it is too simple to use a single parameter to characterize it. Denkena et al. [5] established the form-factor method (also referred to as K-factor method), which more accurately describes the cutting edge micro-geometry, it can be measured by the Alicona Infinite-Focus microscope.

The K-factor of cutting edges are prepared by four-level abrasive medium on the process time. For each section of lapping media, the average cutting edge rounding $\bar{S}$ calculated from Edge Master module to fitting 50 edge contours. At the same time, the length of cutting edge S $\alpha$ and $S \gamma$ are introduced to measure the distance between the ideal sharp cutting edge and flank face and rake face, respectively. The location of the tangent separation of $S \alpha$ and $S \gamma$ largely depends on the fitting area. The K-factor reflects the cutting edge with symmetry and asymmetry. As for asymmetry cutting edge, $(\mathrm{K}<1)$ defined as waterfall hone and $(\mathrm{K}>1)$ defined as trumpet hone. As for symmetry cutting edge defined as $(\mathrm{K}=1)$. Fig. 10 demonstrates the $\mathrm{K}$-factor parameters with four abrasives at process time 20min. It can be seen from the figure that the HSO 1/100 abrasive has the highest removal ability after processing time of 20 minutes, and it is an abrasive that can be considered for large $\mathrm{K}$-factor value. The scope of application of this abrasive will be description in my article. At process parameters, $n_{H}=30 \mathrm{rpm}, n_{R}=100 \mathrm{rpm}$ and $T_{E}=30 \mathrm{~mm}$, the $\mathrm{K}$-factor variation range from 0.9 to 1.2 , because of the symmetrical cutting edge is a commonly prepared parameter in the case of proportional clockwise and counter clockwise rotation. The cutting edge rounding $\bar{S}$ values approximately range from $5 \mu \mathrm{m}$ to $10 \mu \mathrm{m}$ after process time 20 min. The stability of MR mainly comes from the consistency abrasive, while tolerances on the cutting edge geometry and the positioning are somewhat relaxed. The micro-geometry and surface 
finish depends on the size and component of the abrasive particle.

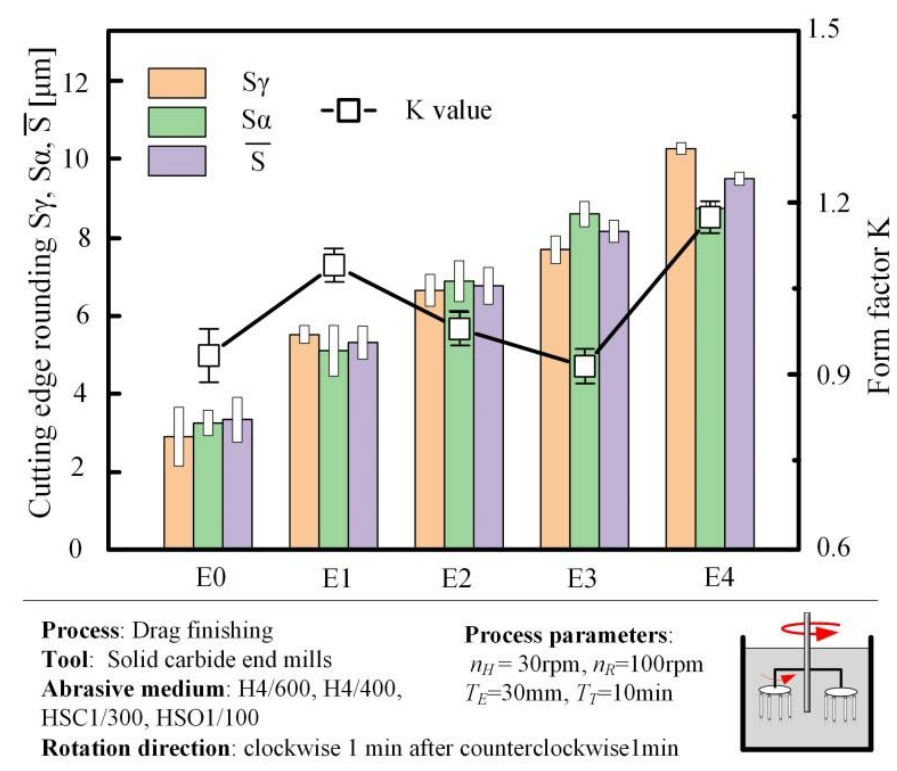

Fig. $10 \mathrm{~K}$-factor by drag finishing for solid carbide end mills

\subsubsection{Cutting edge radius}

According to the research in the previous section, the symmetrical cutting edge is the main goal of this paper. Although, the cutting edge radius $r_{\beta}$ is too simplified, it is still very common in practical applications. The accuracy of cutting edge depiction with a constant circle can be increased by using a higher number of measured points.

The characterization accuracy of the cutting edge radius depends on the selection of the measuring point, which determines the fitting constant circle. Benjamin et al. [45] and Wyen et al. [46] were indicated that fitting algorithm was also a very important factor. The unprepared cutting edges $\left(r_{s}\right)$ is range of $3 \mu \mathrm{m}<r_{s}<5 \mu \mathrm{m}$. The abrasive removal process is shown in Fig. 9. By calculating the mean value and standard deviation of all prepared cutting edge radius, it is possible to evaluate the repeatability of abrasive process. The unprepared cutting edges have some micro defects and grinding marks clearly visible on the rake and flank surfaces. All these edge defects can cause large deviations in the measurement along the cutting edge and lead to reduced cutting stability and tool life. It can be seen from Fig. 11, the cutting edges prepared by four abrasives have prepared different cutting edge radius. Among them, the K3/600 abrasive prepared $r_{\beta}$ is the smallest, so that the normal distribution is dispersed, while the HSO1/100 abrasive obtains the largest $r_{\beta}$, and the measured passivation value fluctuates less, which indicates that the prepared cutting edge is more uniform. The cutting edge radii prepared by $\mathrm{HSC} 1 / 300$ and HSO1/100 abrasives are uniformly distributed, and the mean values are $12,15 \mu \mathrm{m}$ respectively. Because of $\mathrm{SiC}$ has a higher hardness, adding $50 \%$ fine grain $\mathrm{SiC}$ to $\mathrm{K} 3 / 400$ walnut powder has a higher abrasion ability to obtain a larger edge radius. Regarding the application of four abrasives, the abrasives should be tailored specific processing materials and working conditions. 

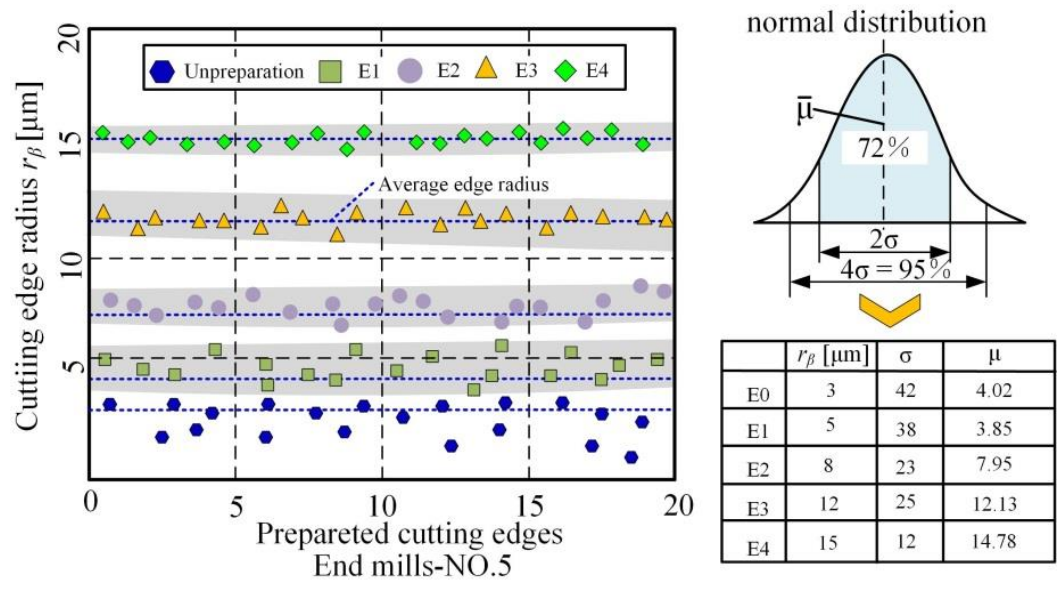

Fig. 11 Cutting edge radius after abrasive process.

\subsection{Surface topography}

\subsubsection{Abrasive process}

As we all know, the preparation of the cutting edge is not only a process of changing the cutting edge geometry, but also a process that modify the surface topography. Fig. 12a depicts a schematic diagram of microscopic damage such as burrs and chipping. The abrasive in DF plays an important role in transferring mechanical energy to the smooth surface reported in [7]. Commonly used abrasives are HSC 1/300, H4/400 and M4/400 is one of the most popular abrasive used for preparation the cutting edge. The surface roughness variation caused by the interaction of solid particles and the cutting edge surface are evaluated by quantifying the $\mathrm{c}$ parameters $R a, R z$ and $R t$. In the $\mathrm{DF}$ finishing process, the abrasion caused by plastic deformation/indentation or micro-cracks in the case of brittle materials is regarded as a hemispherical volume, as shown in Fig. 12b. As shown in Fig. 12c, multiple abrasive particles overlap the cutting edge, resulting in a smooth surface and rounded cutting edge as seen Fig. 12d.
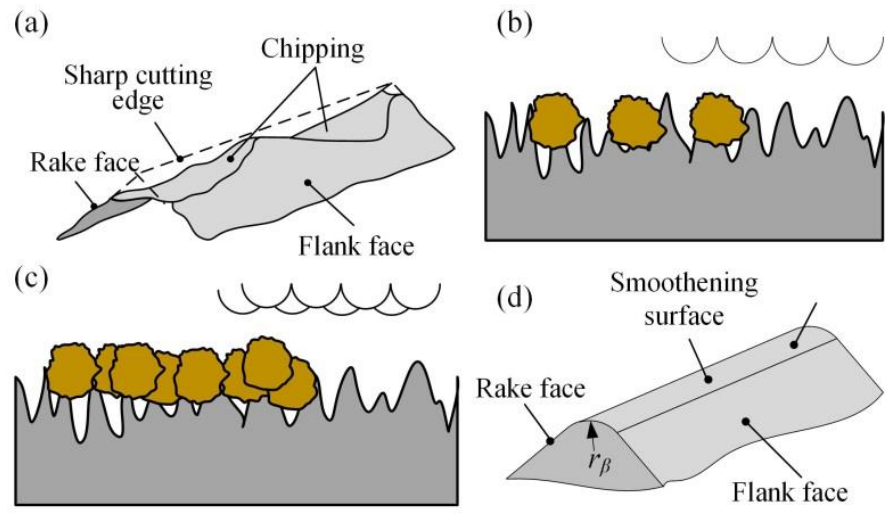

Fig. 12 Schematic diagram of surface smoothing process (a) cutting edge micro-defect after grinding (b) Single abrasive impact (c) multiple, repeated impacts of abrasive impact (d) smooth surface after certain process time

\subsubsection{Cutting edge surface roughness}

This section mainly analyzes the cutting edge surface roughness prepared by the four kinds of abrasives. Denkena et al. [5] studied shown that the surface roughness of the cutting edge is very important for the chip flow, cutting force and temperature distribution in the cutting area, especially for coated cutting tools. Therefore, evaluation of the cutting edge surface quality after 
four kinds abrasive mediums prepared cutting edge roundness is of great significance. In this way, successive cross sections of the cutting edges roughness can be monitored, variation of average roughness $R a, R z$ and $R t$, after drag finishing can be estimated. Out of the large number of experiments conducted, 2D profile of cutting edge is selected relatively stable cutting edge of solid carbide end mill, as presented in Fig. 13. The measurement of the edge chipping has been summarized in Fig. 14. As the size of the larger rounding increases, the chipping $R t$ decreases. The error bars in the figure are represent the standard deviation of the measurements. In order to prove the repeatability of the microscopic geometry, the cutting edge prepared by each abrasive was measured three times.

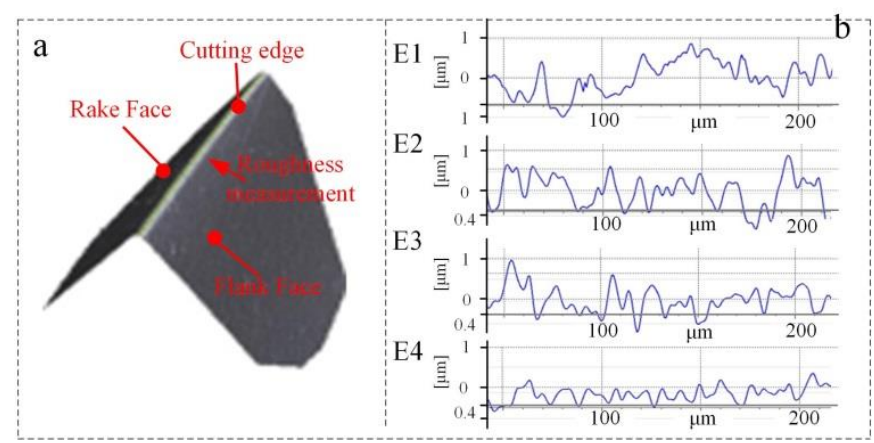

Fig. 13 The cutting edge roughness measurement (a) cutting edge 3D topography; (b) cutting edge 2-D profile

Notably, the roughness $R a$ and $R z$ decrease with different abrasive. Since the removal of material depends on the interaction between the tool and the solid particles of the abrasive, the height and distance between the peaks are significantly decreased. This is due to the solid abrasive is squeezed to remove micro-defects on the cutting edge surface and removes surface peaks in each process. As explained by the previous theory, Uhlmann et al. [14] studied clearly proven the effectiveness of elastic abrasion. The critical values of $R t$ after a processing time of $20 \mathrm{~min}$ are E0: $3.5 \pm 0.1 \mu \mathrm{m}, \mathrm{E} 1: 1.8 \pm 0.85 \mu \mathrm{m}, \mathrm{E} 2: 1.36 \pm 0.53 \mu \mathrm{m}, \mathrm{E} 3: 2.0 \pm 0.53 \mu \mathrm{m}$ and E4: $0.9 \pm 0.53 \mu \mathrm{m}$, respectively. This is due to the removal ability of different abrasive media, include abrasive geometry, composition and kinetic energy vibration and fluctuations in the abrasion process.

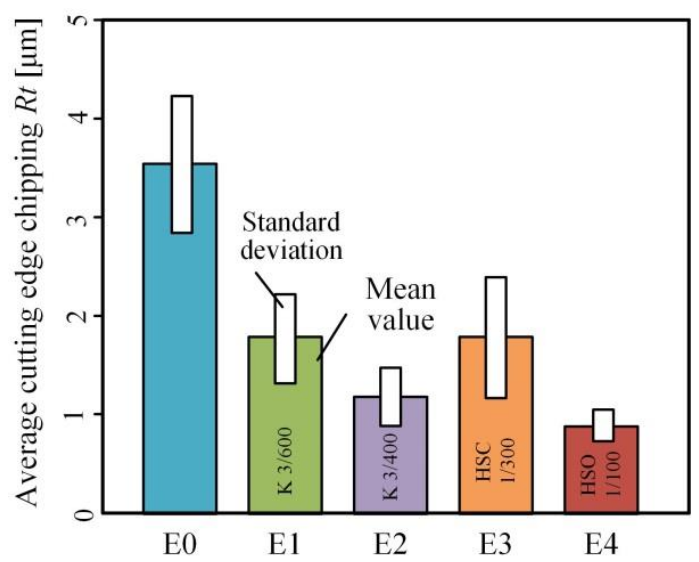

Fig. 14 Average cutting edge chipping $R t$ after drag finishing 


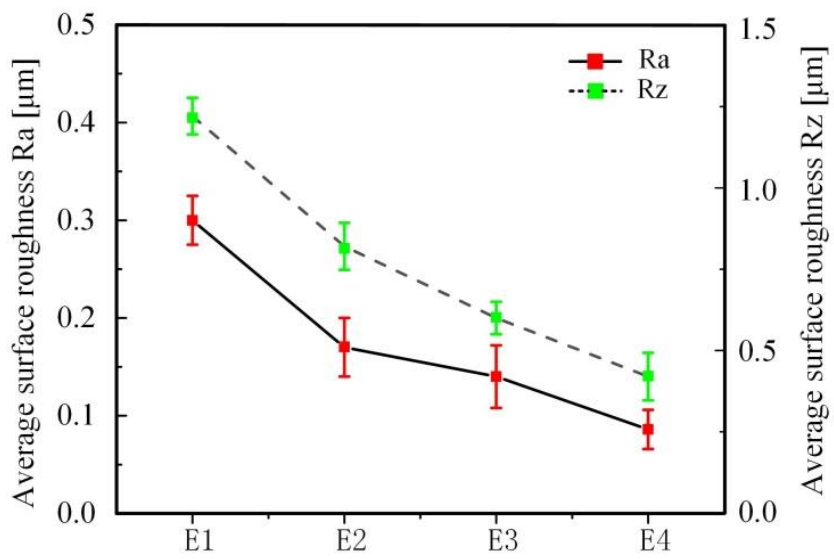

Fig. 1 Cutting edge roughness $R a$ and $R z$

In Fig. 15, the surface roughness of the cutting edge prepared by K3/600, K3/400, HSC 1/300 and HSO 1/100 abrasives are measured. Experimental results show that the abrasive HSO 1/100 mixed $\mathrm{SiO}_{2}$ abrasive $50 \%$ exhibits a smaller average surface roughness $(R a)$. Denkena et al. [2] recognized as the solid carbide end mills was a form of brittleness removal during the cutting edge preparation process, and the surface is smoothed on and around the cutting edge. The surface roughness value of all prepared cutting edges are lower than the unprepared edge. It can be observed that cutting edge roughness decreased by HSC 1/100 abrasive medium preparation. It is due to the larger material removal ability, the micro-defect disappeared with increasing the cutting edge radius and obtained the better surface quality.

\subsubsection{Cutting edge micro-topography}

In order to further analysize the influence of drag finishing on the surface structure of solid carbide end mills, SEM was used to measure the cutting edge morphology using EVO 18 Zeiss. Solid carbide end mills are prepared by four-abrasive in Table 3. As mentioned by Bouzakis et al. [3] and other reletive work Ricardo et al. [29], sharp cutting edge of solid carbide end mills without drag finishing treatment have irregular shapes and micro defect as shown in Fig. 12a. Cutting edge micro-topography describes the surface structure of the cutting edge, as shown in Fig. 16. Corresponding SEM-supported investigations, the rake face and flank face and near the cutting edge were smoothly after HSO 1/100 abrasive prepared E4 group end mills, no obvious signs of micro-breakages and consistent cutting edge micro-geometry was obtained. By observing other abrasives to prepare the cutting edge, the micro morphology of the cutting edge have not been well improved. Comparing K3/600, K3/400 and $\mathrm{HSC} 1 / 300$ abrasives to prepare solid carbide end mills E1, E2 and E3, the cutting edges micro geometry and topography structure are all processed, but the grinding track is still clearly visible on the rake face. As we all know, the roughness of the rake face was greatly significant the chip flow and the quality of cutting edge and thus affects the tool life. This paper researches the process of abrasive to prepare the cutting edges, but for coated tools, it is necessary to combine the tool coating tailor the preparation technics enforce its performance. In the future, the interaction between the cutting edge and the combination tool wear and life will respect to the attention of scientists. 


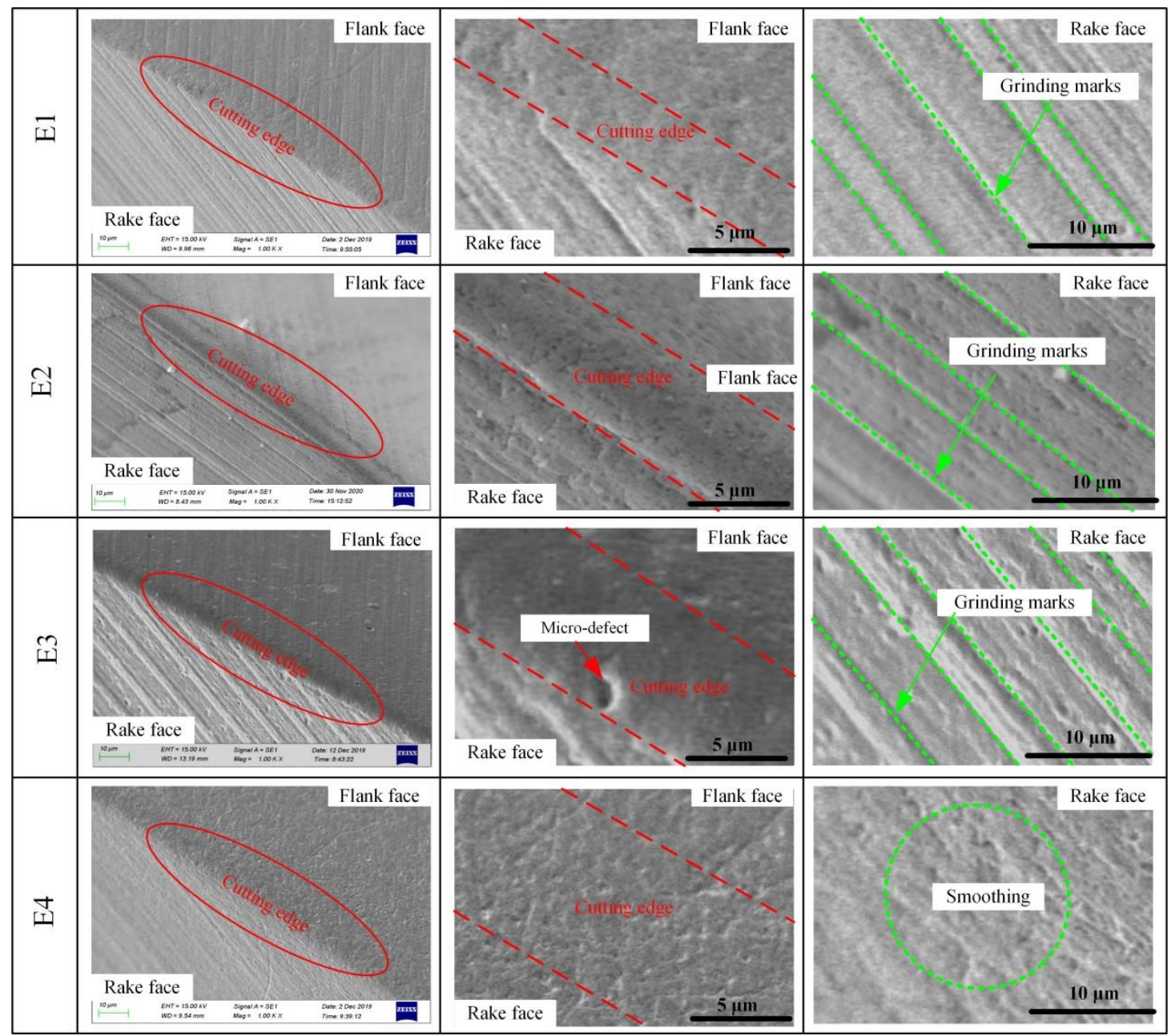

Fig. 16 SEM micro-graphs of four prepared solid carbide end mills

\section{Conclusions}

This article details the cutting edge preparation using four-abrasive medium prepared solid carbide end mills by drag finishing on the mechanism of movement trajectory, material removal, cutting edge characterization, and surface topography. In this work, the efforts were made to analysis four abrasive to prepare the complex solid carbide end mills using drag finishing are listed below:

- The mechanism of material removal and trajectory motion by drag finishing with two-stage planetary motion was obtained. Material removal volume $A_{r}$ uses 4 abrasives (K3/600, K3/400, HSC $1 / 300$ and HSO 1/100 ) to analyze the ability of removal material after process time $20 \mathrm{~min}$.

- Geometric characterization shows the ability of the four-abrasive medium using drag finishing process to produce uncoated solid carbide end mills for different shapes and sizes (average cutting edge rounding $\bar{S}$ ranges from 5 to $10 \mu \mathrm{m}$ ). The K-factor parameters with $\mathrm{S} \alpha, \mathrm{S} \gamma, \mathrm{K}$ and cutting edge rounding $\bar{S}$ were measured by Edge Master Module.

- The abrasive HSO $1 / 100$ has obtained the highest cutting edge radius $r_{\beta}$ with $15 \mu \mathrm{m}$ and the measurement data is analyzed by Gaussian fitting. The smaller the $\sigma$, the more concentrated the edge radius, in other words, more scattered, the variance $u$ determines the size of the cutting edge radius. 
- The cutting edge surface roughness and topography were investigated with four abrasive preparation. The cutting edge chipping $R t$ reflects the cutting edge micro-defect, and determines the quality of the cutting edge. Compared four-abrasive HSO 1/100 has a good abrasive in the preparation of cutting edges, so as to obtain better smoothness.

\section{Acknowledgements}

The authors would like to the Guohong tool system (Wuxi) Co., Ltd for providing the experimental conditions, including drag finishing and cutting experimental devices, tools, workpiece materials, etc.

\section{References}

[1] Li GC, Sun J, Li JF (2014) Modeling and analysis of helical groove grinding in end mill machining. J Mater Process Technol 214: 3067-3076. https:// doi.org/101016/jjmatprotec201407009

[2] Denkena B, Michaelis A, Herrmann M, Pötschke J, Krödel A, Vornberger A, Picker T (2020) Influence of tool material properties on the wear behavior of cemented carbide tools with rounded cutting edges. Wear 15:456-457. https:// doi.org/101016/jwear2020203395

[3] Bouzakis KD, Michailidis N, Skordaris G, Bouzakis E, Biermann D, M'Saoubi R (2012) Cutting with coated tools: coating technologies characterization methods and performance optimization. CIRP Ann 61:703-723. https:// doi.org/101016/jcirp201205006

[4] Karpat Y, Özel T (2008) Mechanics of high speed cutting with curvilinear edge tools. Int J Mach Tools Manuf 48:195-208. https://doi org/101016/jijmachtools200708015

[5] Denkena B, Biermann D (2014) Cutting edge geometries CIRP Ann 63:631-653. https:// doi.org/101016/jcirp201405009

[6] Toenshoff HK, Denkena B (2013) Basics of cutting and abrasive processes. Springer Verlag https://doi101007/978-3-642-33257-9

[7] Uhlmann E, Oberschmidt D, Kuche Y, Löwensteinb A (2014) Cutting edge preparation of micro milling tools. Procedia Cirp 14:349-354. https:// doi.org/101016/jprocir201403083

[8] Uhlmann E, Eulitz A, Dethlefs A (2015) Discrete element modelling of drag finishing. Procedia CIRP 31:369-374. https:// doi.org/101016/jprocir201503021

[9] Gillespie LK (2007) Mass finishing handbook 1st ed New York: Industrial Press

[10] Holzknecht E (2014) Drag finishing displaces manual and robotic grinding \& polishing MFN Metal Finishing News

[11] Nguyen VT, Fang TH (2020) Material removal and wear mechanism in abrasive polishing of $\mathrm{SiO} / \mathrm{SiC}$ using molecular dynamics. Ceram Int 46:21578-21595. https:// doi.org/101016/jceramint202005263

[12] Lee HS, Jeong HD, Dornfeld DA (2013) Semi-empirical material removal rate distribution model for $\mathrm{SiO}_{2}$ chemical mechanical polishing (CMP) processes. Int J Precis Eng 37: 483-490. https:// doi.org/101016/jprecisioneng201212006

[13] Pellegrin DVD, Stachowiak GW (2002) Assessing the role of particle shape and scale in abrasion using 'sharpness analysis': Part I Technique development. Wear 253:1016-1025. https:// doi.org/101016/S0043-1648(02)00232-6

[14] Uhlmann E, Oberschmidt D, Löwenstein A, Kuche Y (2016) Influence of cutting edge preparation on the performance of micro milling Tools. Procedia CIRP 46:214-217. https:// 
doi.org/101016/jprocir201603204

[15] Sooraj VS (2017) On the process and mechanics of rotary elastoabrasive finishing. Mach Sci Technol 21 474-492. https:// doi.org/101080/1091034420171284565

[16] Evans CJ, Paul E, Dornfeld D, Lucca DA, Mullany BA (2003) Material removal mechanisms in lapping and polishing. CIRP Ann 52: 611-633. https:// doi.org/101016/S0007-8506(07)60207-8

[17] Barletta M, Rubino G, Valentini PP (2015) Experimental investigation and modeling of fluidized bed assisted drag finishing according to the theory of localization of plastic deformation and energy absorption. Int J Adv Manuf Technol 77: 2165-2180. https:// doi.org/101007/s00170-014-6620-y

[18] Sundararajan G (1991) A comprehensive model for the solid particle erosion of ductile materials. Wear 149:111-127. https:// doi.org/101016/0043-1648(91)90368-5

[19] Brach RM 1988 Impact dynamics with applications to solid particle erosion Int J Impact Eng 7:37-53. https:// doi.org/101016/0734-743X(88)90011-5

[20] Yang Y Sun TX Yuan YM Gao H Wang XP 2020 Experimental investigation into the effect of abrasive process parameters on the cutting performance for abrasive water jet technology: a case study. Int J Adv Manuf Technol 107:2757-2765. https:// doi.org/101007/s00170-020-05183-3

[21] Fowler G Pashby IR Shipway PH 2009 The effect of particle hardness and shape when abrasive water jet milling titanium alloy Ti6Al4V. Wear 266:613-620. https:// doi.org/101016/jwear200806013

[22] Malkorra I, Salvatore F, Rech J, Arrazola P, Tardelli J, Mathis A (2020) Influence of lubrication condition on the surface integrity induced during drag finishing. Procedia CIRP 87:245-250. https://doi.org/101016/jprocir202002087

[23] Steigerwald JM, Murarka SP, Gutmann RJ (2007) Chemical mechanical planarization -An Introduction. Chemical Mechanical Planarization of Microelectronic Materials. https:// doi.org/ 10.1002/9783527617746.ch1

[24] Holzknecht E 2009 Everything you need to know about mechanical/mass finishing: A workshop on the role of media in mechanical surface finishing. Met Finish 107:27-31. https:// doi.org/101016/S0026-0576(09)80098-6

[25] Nie ZH Liang ZY Wang X Gong J 2018 Evaluation of granular particle roundness using digital image processing and computational geometry. Constr Build Mater 172:319-329. https:// doi.org/101016/jconbuildmat201803246

[26] Weinert K Schneider M 2000 Simulation of tool-grinding with finite element method CIRP Ann 49 253-256. https:// doi.org/101016/S0007-8506(07)62940-0

[27] Lv DJ, Wang YG, Yu X (2020) Effects of cutting edge radius on cutting force tool wear and life in milling of SUS-316L steel. Int J Adv Manuf Technol 111:2833-2844. https:// doi.org/101007/s00170-020-06286-7

[28] Zhou L, Deng B, Peng FY, Yang MH, Yan R (2019) Semi-analytic modeling of cutting forces in micro ball-end milling of NAK80 steel with wear-varying cutting edge and associated nonlinear process characteristics. Int J Mech Sci 169: 105343 https:// doi.org/101016/jijmecsci2019105343

[29] Ricardo GAN, Sommerfeld M (2020) Experimental evaluation of surface roughness variation of ductile materials due to solid particle erosion. Adv Powder Technol 31:3790-3816. https:// 
doi.org/101016/japt202007023

[30] Beaucamp A, Simon P, Charlton P, King C, Matsubara A, Wegener K (2016) Brittle-ductile transition in shape adaptive grinding (SAG) of SiC aspheric optics. Int J Mach Tools Manuf https:// doi.org/101016/jijmachtools201611006

[31] Kato K (1997) Abrasive wear of metals. Tribol Int 30:333-338. https:// doi.org/101016/S0301-679X(96)00063-1

[32] Lin B, Jiang XM, Cao ZC, Huang T, Li KL (2019) Theoretical and experimental analysis of material removal and surface generation in novel fixed abrasive lapping of optical surface. Mater Process Technol 279:1165-1170. https:// doi.org/101016/jjmatprotec2019116570

[33] Hutchings IM, Winter RE (1974) Particle erosion of ductile metals: A mechanism of material removal. Wear 27:121-128. https:// doi.org/101016/0043-1648(74)90091-X

[34] Lu JW, Sargent GA, Conrad H (1995) A Study of the mechanisms of erosion in silicon single crystals using Hertzian fracture tests. Wear 186-187: 105-116. https:// doi.org/101016/0043-1648(95)07128-8

[35] Chiaia B (2001) Fracture mechanisms induced in a brittle material by a hard cutting indenter. Int J Solids Struct 38:7747-7768. https:// doi.org/101016/S0020-7683(01)00117-2

[36] Hutchings IM, Winter RE (1974) Particle erosion of ductile metals: A mechanism of material removal. Wear 27:121-128. https:// doi.org/101016/0043-1648(74)90091-X

[37] Brach RM (1988) Impact dynamics with applications to solid particle erosion. Int J Impact Eng 7:37-53. https:// doi.org/101016/0734-743X(88)90011-5

[38] Sundararajan G (1991) A comprehensive model for the solid particle erosion of ductile materials. Wear 149:111-127. https:// doi.org/101016/0043-1648(91)90368-5

[39] Brach RM, Dunn PF (1992) A mathematical model of the impact and adhesion of microspheres. Aerosol Sci Technol 16:51-64. https:// doi.org/101080/02786829208959537

[40] Sooraj VS, Radhakrishnan V (2013) Elastic impact of abrasives for controlled erosion in fine finishing of surfaces. J Manuf Sci Eng 135: 051019-1. http:// doi.org/101115/14025338

[41] Fowler G, Pashby IR, Shipway PH (2009) The effect of particle hardness and shape when abrasive water jet milling titanium alloy Ti6A14V. Wear 266:613-620. https:// doi.org/101016/jwear200806013

[42] Zhao J, Huang JF, Wang R, Peng HR (2020) Investigation of the optimal parameters for the surface finish of $\mathrm{K} 9$ optical glass using a soft abrasive rotary flow polishing process. J Manuf Processes 49:26-34. http:// doi.org/101016/jjmapro201911011

[43] Sauer K, Witt M, Putz M (2019) Influence of cutting edge radius on process forces in orthogonal machining of carbon fibre reinforced plastics (CFRP). Procedia CIRP 85:218-223. https:// doi.org/101016/jprocir201909042Johnson

[44] Aurich JC, Effgen C, Kirsch B (2016) Cutting edge preparation with elastic bonded superabrasive grinding wheels. CIRP Ann 65:329-332. https:// doi.org/101016/jcirp201604093

[45] Benjamin B, Thilo G (2018) Basic principles for the design of cutting edge roundings. CIRP Ann 67: 73-78. https:// doi.org/101016/jcirp201804019

[46] Wyen CF (2011) Rounded cutting edges and their influence in machining titanium. (PhD-Thesis) ETH Zü rich 


\section{Acknowledgements}

The authors thank the Guohong tool system (Wuxi) Co., Ltd for providing the testing conditions, including SEM and Alicona equipment, etc.

\section{Author information}

\section{Affiliations}

1. School of Mechatronics Engineering and Automation, Shanghai University, Shanghai 200444, China

Dejin Lv, Yongguo Wang $\square$, Xin Yu, Han Chen, Yuan Gao

\section{Contributions}

The first author Lv DJ has been responsible for writing this paper, testing and collecting experimental data, analysis varies abrasives on preparation cutting edge, researching the removal mechanism of abrasives. $\mathrm{Yu} \mathrm{X}$ is responsible for conducting a theoretical model research the abrasive process. Chen $\mathrm{H}$ is responsible for verification, whether as a part of the activity or separate, of the overall reproducibility of results and the cutting edge measurement. Gao Y is responsible for formulation or evolution of overarching research goals and aims. The corresponding author Wang YG is responsible for determining the overall logical structure of the paper and guiding the entire experiment.

\section{Corresponding author}

Correspondence to Y. G. Wang

\section{Ethics declarations}

\section{Ethical approval}

Ethics approval was not required for this research.

\section{Consent to participate}

Not applicable.

\section{Consent for publication}

The authors do agree that the copyright of this paper is transferred to Springer's journal "The International Journal of Advanced Manufacturing Technology" when the paper is accepted for publication.

\section{Competing interests}

The authors declare that they have no conflict of interest.

\section{Additional information}

\section{Publisher's note}

Springer Nature remains neutral with regard to jurisdictional claims in published maps and institutional affiliations. 
Rights and permissions

$\underline{\text { Reprints and Permissions }}$ 